\title{
THE EFFECT OF MENTAL REHEARSAL AND IMAGERY ON MUSIC PERFORMANCE ANXIETY AMONG JUNIOR HIGH SCHOOL STUDENTS
}

\author{
Samuel Agbenyo \\ Department of Music Education, School of Creative Arts, \\ University of Education, Winneba - Ghana \\ E-mail: sagbenyo@go.olemiss.edu
}

Cite this article:

Samuel Agbenyo (2022), The Effect of Mental Rehearsal and Imagery on Music Performance Anxiety among Junior High School Students. Journal of Advanced Research and Multidisciplinary Studies 2(1), 1-8. DOI: 10.52589/JARMS9WZMUBWB

\section{Manuscript History}

Received: 7 Jan 2022

Accepted: 29 Jan 2022

Published: 9 Feb 2022

Copyright $\odot 2022$ The Author(s). This is an Open Access article distributed under the terms of Creative Commons Attribution-NonCommercialNoDerivatives 4.0 International (CC BY-NC-ND 4.0), which permits anyone to share, use, reproduce and redistribute in any medium, provided the original author and source are credited.
ABSTRACT: The purpose of this experimental research was to investigate whether Mental Rehearsal and Imagery (MRI) activities would have effects on Music Performance Anxiety (MPA) among junior high students of Bomigo University Practice School. Subjects $(N=50)$ were taken through a pre-test, a two-day Mental Rehearsal and Imagery (MRI) treatment and a post-test. Their heart rates were recorded by medical experts after each test. The study achieved a ratio level data. Results of a t-test conducted on the two groups revealed $t(11)=4.7, p<.05$, indicating that MRI had a significant influence on MPA. Future research will help measure differences in the extent of effect that MRI may have on Music Performance Anxiety of students at other grades/levels.

KEYWORDS: Mental rehearsal, imagery, music performance anxiety, emotions, heart rates, effect 


\section{INTRODUCTION}

Music Performance Anxiety (MPA), also referred to as stage fright, is a prevalent problem among musicians that may have devastating effects on their well-being and careers (Nagel, 2010). MPA has been conceptualized as a type of social anxiety as well as a Discrete Focal Anxiety (DFA) with normal functioning outside of specific music performance situations (Nicholson, 2015). This problem appears to be a complex phenomenon that results from the interplay of various factors, such as the performer's personal characteristics, the degree of task mastery, the stress of the particular situation and the performers' thoughts about performance (Wilson \& Roland, 2002). However, one important thing, which Wilson and Roland omitted, that could have been useful to readers on this subject matter is the specific identifications or examples of the personal characteristics they referred to in their studies. It is however gratifying to realize an earlier indication by Mor et al. (1995) that personal characteristics associated with MPA include trait anxiety, perfectionism and/or excessive need for controlled neuroticism, introversion, and proneness to social phobia. These personal characteristics, or "basic tendencies" seem to be partly biologically determined (McCrae et al., 2000).

MPA has also been shown to correlate with certain kinds of cognitions, that is, negative selftalk, preoccupation about "not being good enough", concerns about others' negative evaluation, fear of humiliation and catastrophe (believing that minor errors may have catastrophic consequences). Other irrational beliefs, such as the conviction that one must be perfectly competent at musical performance to be a worthwhile person also generate music performance anxiety (Dews \& Williams, 1989; Kenny \& Osborne, 2006). Furthermore, the impact of cognition on emotion is widely acknowledged, and it is particularly emphasized by appraisal theorists who argue that it is not reality per se that elicits emotions, but rather the subject's appraisal, or subjective evaluation of this reality (Frijda et al., 2000; Ortony et al., 1990). Cognitive processes seem to determine the relevance of events as well as elicit and differentiate emotions (Scherer, 2005). That is to say that a performer's thoughts, whether positive or negative about a music performance, can generate emotions which may eventually result in MPA. Frijda (1988) highlights the predictable causal relation that exists between cognition and emotion, claiming that the subjects' appraisals of events inescapably determine the emotions that they will experience. He further explains that such appraisals are done "in response to events that are important to the individual's goals, motives, or concerns" (Frijda, 1988, p. 351).

It appears that music performers tend to develop unique cultural identities with characteristic thoughts, goals, and behaviors different from other groups, with the view to satisfying the prescribed value systems and priorities cherished and upheld by the audience within a given socio-cultural milieu. In effect, the audience of a particular performance context such as the community, the church or as it is in this study, the school, tend to assume the status of 'judges' over the musicians. This way, there is very little recognition (if any) for individuality, independence and personal aesthetic freedom of the performer, thereby heightening emotion and anxiety in the performer (Markus \& Kitayama, 1991; Schwartz, 2006). Contrarily however, it is worthy of note that some cultures do particularly encourage individualism, selfenhancement, or autonomy, whereas others rather praise collectivism, self-transcendence, or embeddedness (Scherer \& Brosch, 2009). Hence, groups or audience differ in the issues they make salient (Oyserman, 2009), issues that become "chronically accessible" (Bargh et al., 1988, p.16), and those that likely impact on the way individuals appraise events (Mesquita \& Karasawa, 2002). Similarly, Damasio argues that culture impacts on the kind of information 
individuals attend to as well as on the way they interpret and evaluate such information; culture also establishes the appropriate emotional responses and consequent musical behavior (Damasio, 1999). Accordingly, it seems that culturally shaped beliefs, goals, and values may lead to "systematic appraisal biases" that will impact on individuals' emotions, negatives of which lead to performance anxiety among musicians. Since the practice school is an academic community with its required performance standards and evaluation system, I consider it in this study as a microcosm cultural context within the macrocosm cultural context of society. Therefore, the foregoing elucidations about music performance anxiety is applicable to the subjects of this experiment.

The purpose of this study was to experiment whether Mental Rehearsal and Imagery (MRI) treatment had effects on Music Performance Anxiety (MPA) among junior high students of the practice (demonstration) school of Bomigo University (pseudonym) in Ghana. It was anticipated that MRI would have no effect on MPA.

\section{METHOD}

\section{Design, Participants and Procedure}

Subjects $(\mathrm{N}=50)$ were randomly sampled from the Demonstration (Practice) Junior High School of Bomigo University in Ghana. With the prior permission of the Headmaster of the school, all students were invited to give voluntary consent to participate in the study by completing a consent form. The first 50 students to turn in their completed and signed consent form were selected for the experiment. The choral music teacher of the school assisted in working with the students.

\section{Pre-test and Post-test}

For a testing instrument, two sight-reading melodies similar to exercises from "Week 13" in McGill and Stevens' 90 Days to Sight Reading Success (2003) were designed, as cited in Lovorn (2016). Each melody was nine measures long, in the key of G major, in simple duple meter, and on the treble staff. Both melodies were made to be of similar difficulty by using the same number of rhythmic changes and the same interval. The instrument is shown in Figure 1 below:

\section{Figure 1: Melodies for pre-test and post-test}

A

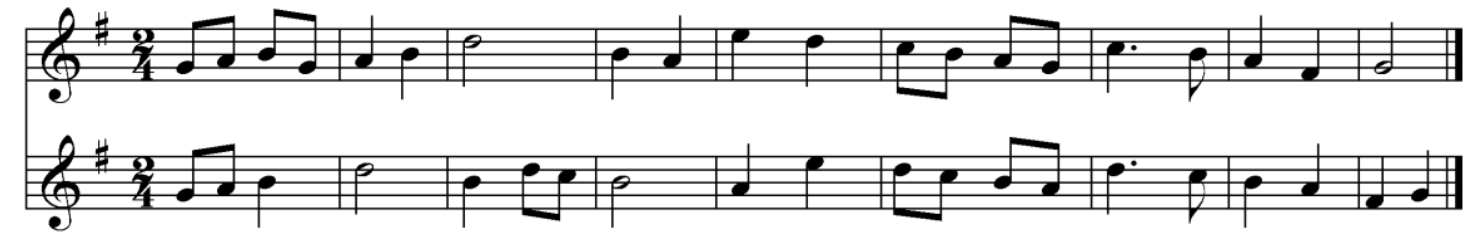

Subject's heart rates were measured on two occasions in the experiment. The first measurement was taken after the pre-test performance and the second measurement was taken after the posttest performance. The post-test was done, following the "Mental Rehearsal and Imagery" activity. Although there are various ways of determining Music Performance Anxiety such as 
brainwave activity and self-report, heart beat rate measurement was chosen for two main reasons. First, to reduce both researcher and subject biasness to the minimum by avoiding the self-report option. Secondly, considering the available medical expert, equipment as well as risk implications, measurement of "heart rates" was the most applicable option for this study. Research suggests that for adults 18 years and older, a normal resting heart rate is between 60 and 100 beats per minute (bpm), depending on the person's physical condition and age. For children of age 6 to 17, the normal resting heart rate is between 70 and $100 \mathrm{bpm}$ (Thurber et al. 2010; LeBlanc et al. 1997). The subjects in this study fell within the first age range (18 years and older). So, their normal heart beat rate was expected to be between 60 and $100 \mathrm{bpm}$ (assuming all other factors are held constant).

Observing the prevailing COVID-19 protocols, performances were done in the main (150 capacity) auditorium of the Music Department in the University, with one teacher and two medical officers being the audience in-person. However, subjects were informed that their performances were being streamed live to the public on zoom. In the pre-test, subjects were given Melody A and allowed 15 minutes to familiarize themselves with the music, followed by a performance. Immediately after the performance, their heart rates were measured and recorded. Later, subjects were randomly divided into two groups: A and B. The experimental group, A ( $\mathrm{n}=25)$ was taught how to do Mental Rehearsal and Imagery (MRI) via zoom but the control group, $B(n=25)$ was not taught how to do MRI. Both groups A and B were subsequently given Melody B and were allowed 15 minutes to familiarize themselves with the music, followed by the post-test. Immediately after performing the post-test, participants' heart rates were measured again and recorded. Two clinical technicians were employed to measure the heart rates of the subjects, using standard electrocardiogram devices in order to maximize reliability.

\section{RESULTS}

The study achieved a ratio level data. Hence, a parametric statistical analysis was done. Prior to intervention, there was not a statistically significant difference for heart rate scores representing MPA levels between the Experimental Group $(M=6.67, S D=5.78)$ and the Control Group $(M=6.79, S D=6.36), p=0.94, d=0.02$. Results of $t$-test on the two groups revealed an obtained $t$-value of 4.8 and critical $t$-value of 2.3. Since $t(11)=4.7, p<.05, \mathrm{H}_{0}$ was rejected. It was concluded that Mental Rehearsal and Imagery (MRI) treatment had a significant effect on Music Performance Anxiety (MPA) among junior high students of the practice (demonstration) school of Bomigo University. Subjects exhibited significant decrease in music performance anxiety as shown is their heart rates following the pre-test and the posttest.

\section{DISCUSSION}

This experimental research sought to determine whether Mental Rehearsal and Imagery (MRI) activities would have effect on Music Performance Anxiety (MPA) among junior high students of the practice (demonstration) school of Bomigo University in Ghana. Results indicated that MRI had a significant effect on MPA, since the beat per minute (bpm) of subjects in the treatment group had reduced significantly, following their exercise of Mental Rehearsal and 
Imagery. Furthermore, although not statistically significant, there were slight disparities between the extent of reductions in the bpm recorded for each participant in the experimental group. This subtle indication attracted attention for thorough discussion. Several factors could have accounted for the differences in the levels of improvement realized in the dependent variable. In the first place, Levy et al. (2005) indicate that there are three aspects of performance anxiety. One is known as stressors which include external factors such as an important audition; internal factors like worrisome thoughts and anxious feelings. The second aspect is distress in the form of internal feeling of tension which is usually caused by the stressor. The third aspect of MPA, known as biological responses, includes 'fight' and 'flight' responses. Similarly, Lehrer (1978) identifies three components of music performance anxiety. These include physiological responses such as increase in heart rate (which has been used in this study), perspiration, blood pressure, sweaty palms, dry mouth and general muscle tension. Another component of MPA is cognitive activity, characterized by inability to think clearly, and lethargy. Besides, there is a third aspect known as behavioral responses which include nervous mannerisms and superstitious or ritual behaviors. All these varieties associated with music performance anxiety suggest that different individuals have different ways of experiencing and exhibiting MPA, depending on their biological compositions and environmental circumstances in which they find themselves at a given point in time. Some of these reasons might have partly accounted for the differences in the heart beat rate per minute of the subjects.

As Alzugaray et al. (2016) posit, music performance anxiety is sometimes attributable to a psychological vulnerability profile and early relationship context whereby factors, such as the study of the previous training context, experiences of previous performances, the type of repertoire to be played and the problem of limited experience, high workload as well as inadequate preparation on the part of the performer, can generate and perpetuate performance anxiety in them. These conditions are likely to be found in various students and in varying magnitudes, hence the differences in bpm. Irrespective of one's major instrumental area, a music student (as in the instance of this study) is susceptible to music performance anxiety since it is usually difficult for a student to combat these multi-faceted phenomena of MPA. Performance anxiety is not a problem limited to any specific category of musicians, neither is it solely associated with a particular musical genre. Perdomo-Guevara (2014) argues that it affects all persons across all musical genres. In his conceptualization of MPA as a type of social anxiety, which is even associated with normal functioning outside of specific music performance situations, Nicholson et al. (2015) explain MPA as a phenomenon not attributable to staging alone but having unique and overlapping components, experienced both on the performance stage and in social settings of the musician. This argument confirms an earlier view of Damasio (1999), describing MPA as a culturally-induced problem.

It is also important to indicate that the various categories, facets and factors identified regarding MPA may not exist and impact insolation. Rather, an individual performer could be affected by combinations of these categories and aspects of music performance anxiety. For instance, a student-performer may develop MPA as a result of inadequate preparation and fear of negative judgment by the assessors/audience. What is imperative, therefore, is for music teachers and researchers to intensify the development of effective coping and treatment strategies for the problem. Just as there are several causes and manifestations of Music Performance Anxiety in different individuals (Boucher \& Ryan, 2011), so must there be varying measures for coping with, and treating the phenomena in different persons. For this reason, Nagel et al. (1989) 
suggest the need to combine both physical and cognitive therapeutic measures in the treatment of music performance anxiety, emphasizing that this is a very effective means of lowering debilitating anxiety in various music performers. Also, studies indicate that there have been several strategies in use to combat Music Performance Anxiety over the years. These include the use of "Free improvisation" (Allen, 2011), "Acceptance and Commitment Therapy - ACT" (Juncos \& Markman, 2015), and performers" "rituals of separation from everyday life" (Geeves et al., 2019). According to Herrera and Cremades (2014), one other strategy found to be very effective in minimizing MPA among university piano students in the state of Chihuahua, Mexico is daily practical "memorization strategies" that embodies aural, kinesthetic and visual memorization. This strategy is directly related to the experimental strategy, Mental Rehearsal, in this study.

Cisek and Kalaska (2004) define Mental Rehearsal as a behavior modeling technique, based on social cognitive theory which argues that observational learning is "largely an information processing activity in which information about the structure of behavior and about environmental events is transformed into symbolic representations that serve as guides for action" (Bandura, 1986, p. 51). According to Virtue (1996), the first record of Mental Rehearsal dates back to 60,000 B.C., when hunters from the ice age painted pictures of animal prey on their wall. They would then throw spears at these paintings, visualizing a successful hunt. The ancient Egyptians also believed that diseases could be cured by the visualization of perfect health (p. 210). In this study, the term Mental Rehearsal is used in relation to practicing musical activities in one's mind, without using an instrument. It requires that the musician (subject) practices the given music "mentally" and subsequently produces it "physically" on the instrument. In the experiment, subjects rehearsed their musical pieces mentally for two days and then performed the music on their respective instruments. Similarly, mental imagery serves a significant adaptive function in the preparation of action and coping with change. According to Marks (1999), mental practice, which employs subjectively experienced images of future events and explores how these events might be influenced by behavioral intervention, enables the experiencer's future actions to be more effectively targeted towards their goals.

Finally, although Hodges (2020) confirms the usage of Mental Rehearsal and Imagery "for long as an effective learning strategy" and cited its usefulness to Arthur Rubinstein (p. 251), he is silent on the application of this strategy to the management of Music Performance Anxiety. Furthermore, several other studies have mentioned the use of MRI for successful music memorization (Broomhead et al., 2018; Coffman, 1990; Connolly \& Williamon, 2004; Kristeva et al., 2003; Pascual-Leone et al., 2003; Pascual-Leone et al., 1995; Rose \& Rose, 1985). However, none of these studies mentioned the application of MRI as a strategy for addressing MPA, suggesting some level of novelty in the results of this experiment. There is a need to conduct several other empirical studies on the use of Mental Rehearsal and Imagery as a treatment of Music Performance Anxiety, to determine its conventionality. Besides, studies on the use of MRI for addressing MPA will help compare findings with similar experiments on different age (grade) groups. Also, more research will indicate the extent of differences that may exist in the MRI treatment of MPA between solo performers and ensemble performers. Meanwhile, all music teachers can apply this treatment in their routine work with their students irrespective of what their major may be. 


\section{REFERENCES}

Allen, R. (2011) Free improvisation and performance anxiety among piano students. Journal of Psychology of Music.41(1) 75-88.

Alzugaray, F. J. Z., et al. (2016). Kenny Music Performance Anxiety Inventory: Confirmatory factor analysis of the Spanish version. Journal of Psychology of Music, 44(3) 340-352

Bandura, A. (1993). Perceived self-efficacy in cognitive development and functioning. Educational psychologist, 28(2), 117-148.

Bargh, J. A., Lombardi, W. J., \& Higgins, E. T. (1988). Automaticity of chronically accessible constructs in person $\times$ situation effects on person perception: It's just a matter of time. Journal of Personality and Social Psychology, 55(4), 599.

Biasutti, M. \& Concina, E. (2014). The Role of Coping Strategy and Experience in Predicting Music Performance Anxiety. Musicae Scientiae, 10(11), 1-14.

Boucher, H., \& Ryan, C. A. (2011). Performance stress and the very young musician. Journal of esearch in Music Education, 58(4), 329-345.

Broomhead, P., et al. (2018). The Effects of a Teacher-Directed Preperformance Routine on Expressive Performance Mindset. Bulletin of the Council for Research in Music Education, 2(15) 57-74.

Chamorro-Premuzic, T., Swami, V., Furnham, A., \& Maakip, I. (2009). The big five personality traits and uses of music: A replication in Malaysia using structural equation modeling. Journal of Individual Differences, 30(1), 20-27.

Cisek, P., \& Kalaska, J. F. (2004). Neural correlates of mental rehearsal in dorsal premotor cortex. Nature, 431(7011), 993-996.

Coffman, D. D. (1990). Effects of mental practice, physical practice, and knowledge of results on piano performance. Journal of Research in Music Education, 38(3), 187-196.

Connolly, C., \& Williamon, A. (2004). Mental skills training. Musical excellence: Strategies and techniques to enhance performance, 5(1), 221-245.

Frijda, N. H. (2017). The laws of emotion. Psychology Press.

Frijda, N. H., Manstead, A. S., \& Bem, S. E. (2000). Emotions and belief: How feelings influence thoughts. Cambridge University Press.

Geeves, A. M., et. al. (2019. Seeing Yellow: 'Connection' and Routine in Professional Musicians' Experience of Music Performance. Journal of Psychology of Music.10(11), $1-19$

Herrera, M., \& Cremades, R. (2014). Memorization in Piano Students: A Study in the Mexican Context. Musicae Scientiae, 10(1), 1-16.

Hodges, D. (2020). Music in the human experience: An introduction to music psychology (2nd ed.). Routledge.

Juncos, D. G \& Markman, E. J. (2015). Acceptance and Commitment Therapy for the treatment of music performance anxiety: A single subject design with a university student. Journal of Psychology of Music, 10(1), 1-18.

Kenny Music Performance Anxiety Inventory: Confirmatory factor analysis of the Spanish version. Journal of Psychology of Music, 44(3), 340-352.

Kristeva, R., Chakarov, V., Schulte-Mönting, J., \& Spreer, J. (2003). Activation of cortical areas in music execution and imagining: a high-resolution EEG study. NeuroImage, 20(3), 1872- 1883.

LeBlanc, A., Jin, Y. C., Obert, M., \& Siivola, C. (1997). Effect of audience on music performance anxiety. Journal of research in music education, 45(3), 480-496. 
Levy, S., Rosen, D., \& Sataloff, R. (2005). Performance anxiety. Professional voice, 2(1), 585590.

Marks, D. F. (1999). Consciousness, mental imagery and action. British journal of psychology, 90(4), 567-585.

Markus, H. R., \& Kitayama, S. (1991). Culture and the self: Implications for cognition, emotion, and motivation. Psychological review, 98(2), 224.

McCrae, R. R., Costa Jr, P. T., Ostendorf, F., Angleitner, A., Hřebíčková, M., Avia, M. D., ... \& Smith, P. B. (2000). Nature over nurture: temperament, personality, and life span development. Journal of personality and social psychology, 78(1), 173.

Mesquita, B., \& Karasawa, M. (2002). Different emotional lives. Cognition \& Emotion, 16(1), 127-141.

Mor, S., Day, H. I., Flett, G. L., \& Hewitt, P. L. (1995). Perfectionism, control, and components of performance anxiety in professional artists. Cognitive Therapy and Research, 19(2), 207-225.

Nagel, J. J. (2010). Treatment of music performance anxiety via psychological approaches: A review of selected CBT and psychodynamic literature. Medical Problems of Performing Artists, 25(4), 141-148.

Nagel, J. J., et al. (1989). Cognitive-Behavioral Treatment of Musical Performance Anxiety. Journal of Psychology of Music, 17(1), 12-2.

Nicholson, D. R., Cody, M. W., \& Beck, J. G. (2015). Anxiety in musicians: On and off stage. Psychology of Music, 43(3), 438-449.

Nicholson, D. R., et al. (2015). Anxiety in musicians: On and off stage. Journal of Psychology of Music, 43(3) 438-449.

Ortony, A., Clore, G. L., \& Collins, A. (1990). The cognitive structure of emotions. Cambridge university press.

Oyserman, D. (2009). Identity-based motivation and consumer behavior. Journal of Consumer Psychology, 19(3), 276-279.

Perdomo-Guevara, E. (2014). Is Music Performance Anxiety Just an Individual Problem? Exploring the Impact of Musical Environments on Performers' Approaches to Performance and Emotions. Journal of Psychomusicology, 24(1), 66-74.

Rose, C., \& Rose, D. (1985). Accelerated learning. London: Topaz.

Scherer, K. R., \& Brosch, T. (2009). Culture-specific appraisal biases contribute to emotion dispositions. European Journal of Personality: Published for the European Association of Personality Psychology, 23(3), 265-288.

Schwartz, S. (2006). A theory of cultural value orientations: Explication and applications. Comparative sociology, 5(2-3), 137-182.

Stoeber, J., \& Eismann, U. (2007). Perfectionism in young musicians: Relations with motivation,effort, achievement, and distress. Personality and Individual Differences, 43(8), 2182- 2192.

Thurber, M. R., Bodenhamer-Davis, E., Johnson, M., Chesky, K., \& Chandler, C. K. (2010). Effects of heart rate variability coherence biofeedback training and emotional management techniques to decrease music performance anxiety. Biofeedback, 38(1), 2840.

Virtue, D. (1996). I'd change my life if I had more time. United States: Hay House.

Wilson, G. D., \& Roland, D. (2002). Performance anxiety. The science and psychology of music performance: Creative strategies for teaching and learning, 10(7), 47-61. 